G. Bernardinis, F. Borghetti, V. Ferragina, A. Fornasari, U. Gatti, P. Malcovati, F. Maloberti: "A wide-band 280-MHz four-path time-interleaved bandpass sigma-delta modulator"; IEEE Transactions on Circuits and Systems I: Regular Papers, Vol. 53, Issue 7, July 2006, pp. 1423-1432.

(C)20xx IEEE. Personal use of this material is permitted. However, permission to reprint/republish this material for advertising or promotional purposes or for creating new collective works for resale or redistribution to servers or lists, or to reuse any copyrighted component of this work in other works must be obtained from the IEEE. 


\section{A Wide-Band 280-MHz Four-Path Time-Interleaved Bandpass Sigma-Delta Modulator}

Gabriele Bernardinis, Member, IEEE, Fausto Borghetti, Vincenzo Ferragina, Andrea Fornasari, Umberto Gatti, Member, IEEE, Piero Malcovati, Senior Member, IEEE, and Franco Maloberti, Fellow, IEEE

\begin{abstract}
This paper describes a 0.35- $\mu \mathrm{m}$ CMOS fourth-order bandpass analog-digital sigma-delta $(\Sigma \Delta)$ modulator for wideband base stations receivers. The modulator, based on a time-interleaved four-path architecture, achieves an equivalent sampling frequency of $280 \mathrm{MHz}$, although the building blocks operate at only $70 \mathrm{MHz}$. In measurements, the prototype chip achieves a dynamic range of $72 \mathrm{~dB}$ (12 bits of resolution) with a signal bandwidth of 4.375 $\mathrm{MHz}$ centered around an intermediate frequency of $70 \mathrm{MHz}$. The measured spurious-free dynamic range is $69 \mathrm{~dB}$. The $\Sigma \Delta$ modulator dissipates $480 \mathrm{~mW}$ from a $3.3-\mathrm{V}$ supply, including voltage reference buffers and output pads with high-driving capabilities, and occupies $20 \mathrm{~mm}^{2}$ of silicon area.
\end{abstract}

Index Terms-Calibration technique, time-interleaved ADC, sigma-delta $(\Sigma \Delta)$ modulator.

\section{INTRODUCTION}

$\mathbf{T}$ HE TREND in the receiver chains used in modern telecommunication architectures is to move the analog-to-digital converter (ADC) as close as possible to the antenna. This allows performing most of the signal processing in the digital domain, thus gaining in reconfigurability and programmability. A typical superheterodyne architecture requires to directly digitize the intermediate frequency (IF). The resulting specifications for the integrated ADC are challenging, especially in terms of speed, signal-to-noise ratio (SNR), dynamic range (DR), and spurious-free dynamic range (SFDR) [1]. System-level studies show that an optimal receiver uses IF frequencies around several tens of megahertz (typically $60-80 \mathrm{MHz}$ ) [2], [3]. With a conventional approach, this leads to the use of a clock frequency for the ADC significantly higher than the IF (for example, twice the IF, i.e., $120-160 \mathrm{MHz}$ ), relying on the fast

Manuscript received July 13, 2005; revised November 10, 2005. This work was supported in part by MEDEA+ in the frame of Project ANASTASIA+ A510. This paper was recommended by Associate Editor T. B. Tarim.

G. Bernardinis was with the Department of Electronics, University of Pavia, 27100 Pavia, Italy. He is now with International Rectifier, El Segundo, CA 90245 USA (e-mail: gbernar1@irf.com).

F. Borghetti was with the Department of Electrical Engineering, University of Pavia, 27100 Pavia, Italy. He is now with the Integrated Optical Sensor Group, Istituto Trentino di Cultura/Istituto Ricerca Scientifica e Tecnologica (ITC-IRST), 38050 Povo, Trento, Italy (e-mail: fausto.borghetti@unipv.it; borghetti@itc.it).

V. Ferragina and F. Maloberti are with the Department of Electronics, University of Pavia, 27100 Pavia, Italy (e-mail: vincenzo.ferragina@unipv.it; franco. maloberti@unipv.it).

P. Malcovati is with the Department of Electrical Engineering, University of Pavia, 27100 Pavia, Italy (e-mail: piero.malcovati@unipv.it).

A. Fornasari was with the Department of Electronics, University of Pavia, 27100 Pavia, Italy. He is now with the Austriamicrosystems Italy S.R.L., 27100 Pavia, Italy (e-mail: andrea.fornasari@austriamicrosystems.com).

U. Gatti is with the Siemens Mobile Communications S.p.A., 20092 Cinisello Balsamo, Milan, Italy (e-mail: umberto.gatti@ siemens.com).

Digital Object Identifier 10.1109/TCSI.2006.875191

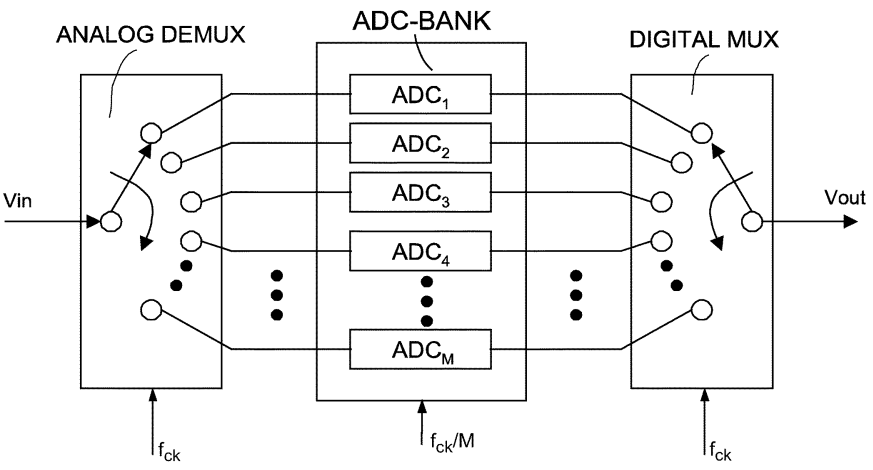

Fig. 1. Block diagram of a time-interleaved ADC.

response of state-of-the-art CMOS processes, or on the use of bipolar or BiCMOS technologies. However, the use of these technologies increases costs and makes the design of the basic blocks of the converter extremely difficult.

In the literature, solutions based on a double-sampling technique with two nonoverlapping clock phases have been reported [4], [5], which achieve good performance. However, the speed requirements for their building blocks is a critical issue.

The approach used in this paper is the use of time-interleaving. Several ADCs operate in parallel, using different clock phases [6], as shown in Fig. 1. An analog demultiplexer selects sequentially each ADC, which therefore operates at the low speed. The digital multiplexer interleaves the digital output of the ADCs, thus producing the overall A/D conversion result. Any type of ADC can be used, including sigma-delta $(\Sigma \Delta)$ modulators [7]. Each of them operates at a clock frequency $f_{\mathrm{ck}} / N$, where $f_{\mathrm{ck}}$ is the overall sampling frequency and $N$ is the number of channels (or paths) used. The speed requirements for each converter are therefore relaxed by a factor $N$. This is achieved at the expense of spurious tones due to offset and gain mismatches among the different ADCs. This tones if necessary can be canceled with suitable calibration techniques [8].

In this kind of application, a bandpass ADC turns out to be more effective than its low-pass counterpart. With a low-pass ADC the requirement to use an IF of several tens of megahertz while the signal band is only few megahertz around the IF would result in a waste of resources. Also, the use of high-speed converters with a low-pass transfer function extended over high Nyquist zone is not effective. By contrast, a bandpass transfer function centred at the desired IF enables converting into the digital domain only the band around the IF and reduces the in-band thermal noise requirements (the thermal noise outside the signal band is rejected in the digital domain). 


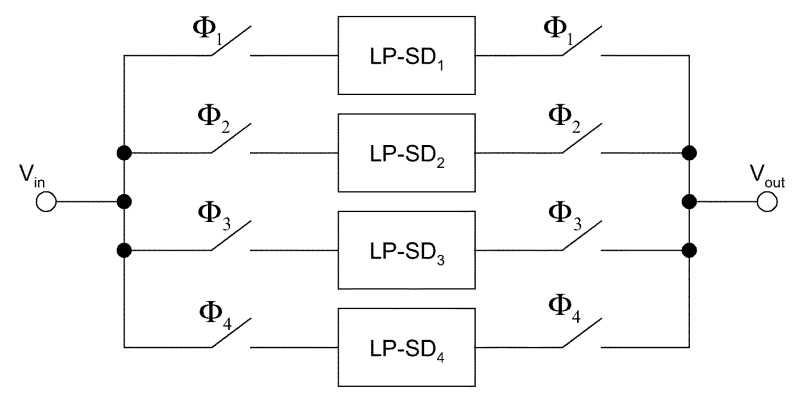

(a)

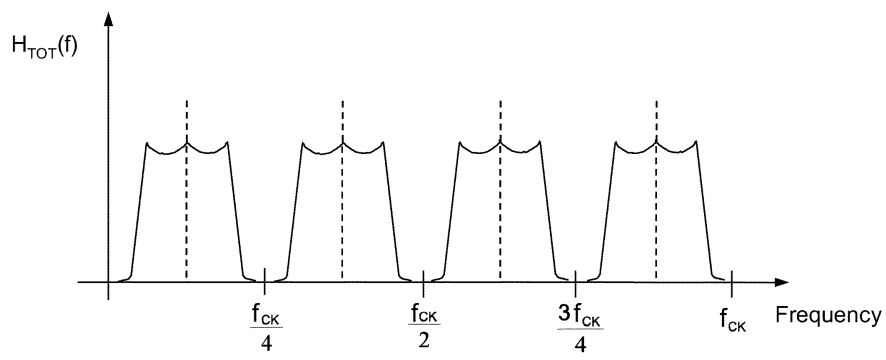

(b)

Fig. 2. (a) Four-path $\Sigma \Delta$ modulator and i (b) ts NTF.

This paper describes the design of a four-path fourth-order bandpass $\Sigma \Delta$ modulator whose performance satisfies the requirements of a typical $3 \mathrm{G}$ multi-carrier wireless receiver [9], [10]. The signal bandwidth of the modulator is $4.375 \mathrm{MHz}$ centered around an IF of $70 \mathrm{MHz}$. The overall clock frequency is $280 \mathrm{MHz}$, while each path works at $70 \mathrm{MHz}$. The paper is organized as follows. Section II describes the four-path $\Sigma \Delta$ modulator. Section III discusses the switched-capacitor (SC) implementation, while details on the design of building blocks are presented in Section IV. Finally, Section V reports experimental results obtained from an integrated prototype.

\section{Multi-Path $\Sigma \Delta$ Modulator}

$\Sigma \Delta$ modulators used in a multi-path architecture obtain a bandpass response by using low-pass $\Sigma \Delta$ modulators in the single paths [Fig. 2(a)]. This feature simplifies the design of the ADC: low-pass $\Sigma \Delta$ modulators are inherently less sensitive to component mismatches than bandpass modulators and, therefore, dynamic range and stability become less critical issues.

It is known that a multiple-path circuit achieves, in the sampled-data domain, a $z$ to $z^{N}$ transformation. Therefore, if $H_{p}(z)$ is the transfer function of the single path, the total transfer function becomes

$$
H_{\mathrm{TOT}}=\frac{V_{\mathrm{out}}(z)}{V_{\mathrm{in}}(z)}=H_{p}\left(z^{N}\right) .
$$

If $H_{p}(z)$ is a low-pass filter, the response folds at multiples of $f_{\text {ck }} / N$, as shown in Fig. 2(b), thus leading, for $N=4$, to a bandpass transfer function centered around $f_{\mathrm{ck}} / 4$.

Moreover, the $z$ to $z^{N}$ mapping given by the multi-path topology multiplies by $N$ the order of the transfer functions. Thus, an $N$-path architecture achieves the same signal-to-noise-and-distortion ratio (SNDR) of single-path architectures with lower order loop filter with benefits in terms
TABLE I

FOUR-PATH BANDPASS $\Delta \Sigma$ MODULATOR FOR UMTS BASE TRANSCEIVER STATIONS

\begin{tabular}{|l|c|}
\hline \multicolumn{1}{|c|}{ Parameter } & Value \\
\hline Clock frequency & $280 \mathrm{MHz}$ \\
\hline Center frequency & $70 \mathrm{MHz}$ \\
\hline Bandwidth & $4.375 \mathrm{MHz}$ \\
\hline Dynamic range & $72 \mathrm{~dB}$ \\
\hline Resolution & $12 \mathrm{bits}$ \\
\hline Spurious-free dynamic range & $70 \mathrm{~dB}$ \\
\hline Quantizer & 9 levels \\
\hline Order (of each path) & 4 \\
\hline
\end{tabular}

of stability and complexity. Finally, an overall clock frequency which is an integer number of times the IF simplifies the demodulation operation.

Table I summarizes the ADC specifications for a converter used in the base transceiver station for third-generation mobile communication standards. The four paths of the ADC operate with a 70-MHz clock while the overall clock frequency is $280 \mathrm{MHz}$. The required resolution is $12 \mathrm{bit}$. With a fourth-order modulator it is necessary to use a nine-level quantizer.

The noise transfer function (NTF) is the first concern of the modulator design. Then, it is necessary to verify if the obtained signal transfer function (STF) is flat in the signal band. As already mentioned the $N$-path architecture achieves the band-stop NTF using in the single paths a low-pass $\Sigma \Delta$ modulator that features a high-pass NTF response. Therefore, the main focus is on the design of a suitable low-pass $\Sigma \Delta$ modulator.

All the architectures used for $\Sigma \Delta$ modulator implementation have the quantizer in a feedback loop which features a sampled-data transfer function $H(z)$. The STF is $H(z) /[1+H(z)]$ while the NTF is $1 /[1+H(z)]$. The required noise specifications and the quantization noise level (taking into account the resolution of the quantizer, nine levels in the considered case) lead to a suitable filtering mask for the NTF. Using a nine-level quantizer ensures stability of the modulator and limits idle tones, being the power of the quantization noise limited. For this design we obtain a high-pass NTF mask with a stop-band limit at $2.1875 \mathrm{MHz}$, in-band attenuation of $85 \mathrm{~dB}$, and ripple of $0.5 \mathrm{~dB}$. The transition band spans from 2.1875 to $10 \mathrm{MHz}$. Filters that fulfill these specifications have been designed using the elliptical, Chebyshev and Butterworth approximation. As expected, the most effective solution is the elliptical filter. The obtained filter order is four with two couples of complex conjugate poles and two couples of complex conjugate zeros located at 0.95 and $2.1875 \mathrm{MHz}$, respectively. Assuming the use of a sampling frequency equal to $70 \mathrm{MHz}$, the NTF in the $z$-domain is

$$
\begin{aligned}
& \operatorname{NTF}(z) \\
& \quad=\frac{1-3.9571 z+5.91445 z^{2}-3.9571 z^{3}+z^{4}}{1-0.949 z+1.0511 z^{2}-0.2909 z^{3}+0.2544 z^{4}}
\end{aligned}
$$

leading to the response of Fig. 3. 


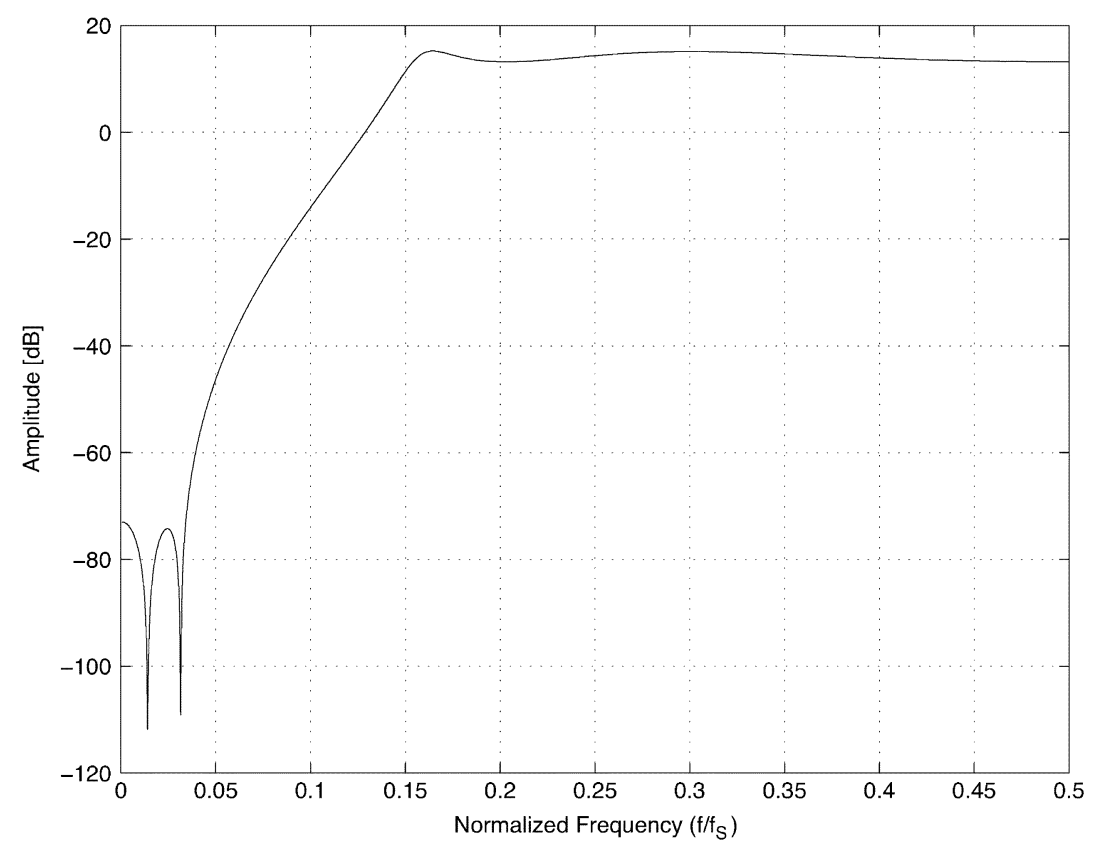

Fig. 3. NTF obtained with the elliptical filter.

The $z$-domain loop transfer function $H(z)$ that allows us to obtains $\operatorname{NTF}(z)$ results from

$$
\begin{aligned}
\operatorname{NTF}(z) & =\frac{P_{e}(z)}{Q_{e}(z)}=\frac{1}{1+H(z)} \\
H(z) & =\frac{Q_{e}(z)-P_{e}(z)}{P_{e}(z)}
\end{aligned}
$$

that requires an architecture with a suitable number of degrees of freedom for the circuit implementation. The use of the polynomials of (2) in (3) leads to seven different coefficients. Conventional architectures, like cascade of resonator in feedback, cascade of resonator in feedforward or cascade of integrators do not provide enough design parameters. It is therefore necessary to add extra elements for increasing the number of degrees of freedom. The nonconventional topology of Fig. 4 uses two extra feedforward branches and allows us to synthesize the required transfer function. The scheme includes two resonators whose structure is shown in the insets of Fig. 4. Behavioral simulations on this architecture have shown that a mismatch as large as $5 \%$ of the coefficient values (including the additional feedforward paths) does not degrade the modulator performance.

A critical feature of this topology is that it allows only one clock period delay $\left(z^{-1}\right)$ between the output of the third integrator and the output of the digital-analog converter (DAC). The sampled-data circuit implementation will distribute the delay associated to the two phases among the cascaded blocks but the simultaneous settling of a pair of cascaded integrators cannot be avoided. In particular, only half clock period is assigned to the cascade of the third and fourth integrator, which therefore have to settle concurrently, while another half clock period is reserved to the comparators and the DAC.

\section{SC IMPLEMENTATION}

Fig. 5 shows the SC implementation of the resonators of Fig. 4. The matching of capacitors affects the accuracy of coef- ficients and, in turn, influences the accuracy of poles and zeros. The required minimum standard deviation of the mismatch $\sigma_{C \text {,min }}$ determines the size of the unity capacitance. As already mentioned, simulations performed with the toolbox described in [11] show a limited sensitivity to coefficient values: the required matching accuracy is as large as $5 \%$. The variance of capacitance mismatch is given by $\sigma_{C}=A_{C} /\left(\sqrt{C / C_{\mathrm{ox}}}\right)$. For the used process $A_{C}=0.012$ and $C_{\mathrm{ox}}=0.86 \mathrm{fF} / \mu \mathrm{m}^{2}$; therefore, in order to fulfill the coefficient matching requirements, considering also finite gain and bandwidth of the operational amplifiers, the unity capacitance can be $250 \mathrm{fF}$. This value is low but the large spread of coefficients would lead to a significant capacitance values. In order to limit the capacitance spread the circuit uses for the most critical elements (the feedback capacitors in the resonators) $\mathrm{T}$-structures with equivalent capacitance $C_{T}$ given by

$$
C_{T}=\frac{C_{T 1} C_{T 3}}{C_{T 1}+C_{T 2}+C_{T 3}} .
$$

The transfer function of the resonator of Fig. 5 is

$$
H_{R}(z)=\frac{C_{2} C_{1} z^{-1 / 2}}{C_{f 2} C_{f 1}\left(1-z^{-1}\right)^{2}+z^{-1} C_{2} C_{T}}
$$

giving the pair of poles

$$
z_{1,2}=e^{ \pm j \vartheta} ; \quad \vartheta=\operatorname{acos}\left(1-\frac{C_{2} C_{T}}{2 C_{f 1} C_{f 2}}\right)
$$

where $\vartheta$ is the angular position on the unit circle of the $z$-plane. The resonance frequency of the circuit is $\omega_{0} f_{s} \cdot \vartheta$. For this design the value of the angular positions of the poles of the two resonators are $\vartheta_{1}=0.013571 \mathrm{rad}$ and $\vartheta_{2}=0.03125 \mathrm{rad}$.

Fig. 6 shows the schematic of the quantizer which is also used for the addition of the four signals coming from the resonator 


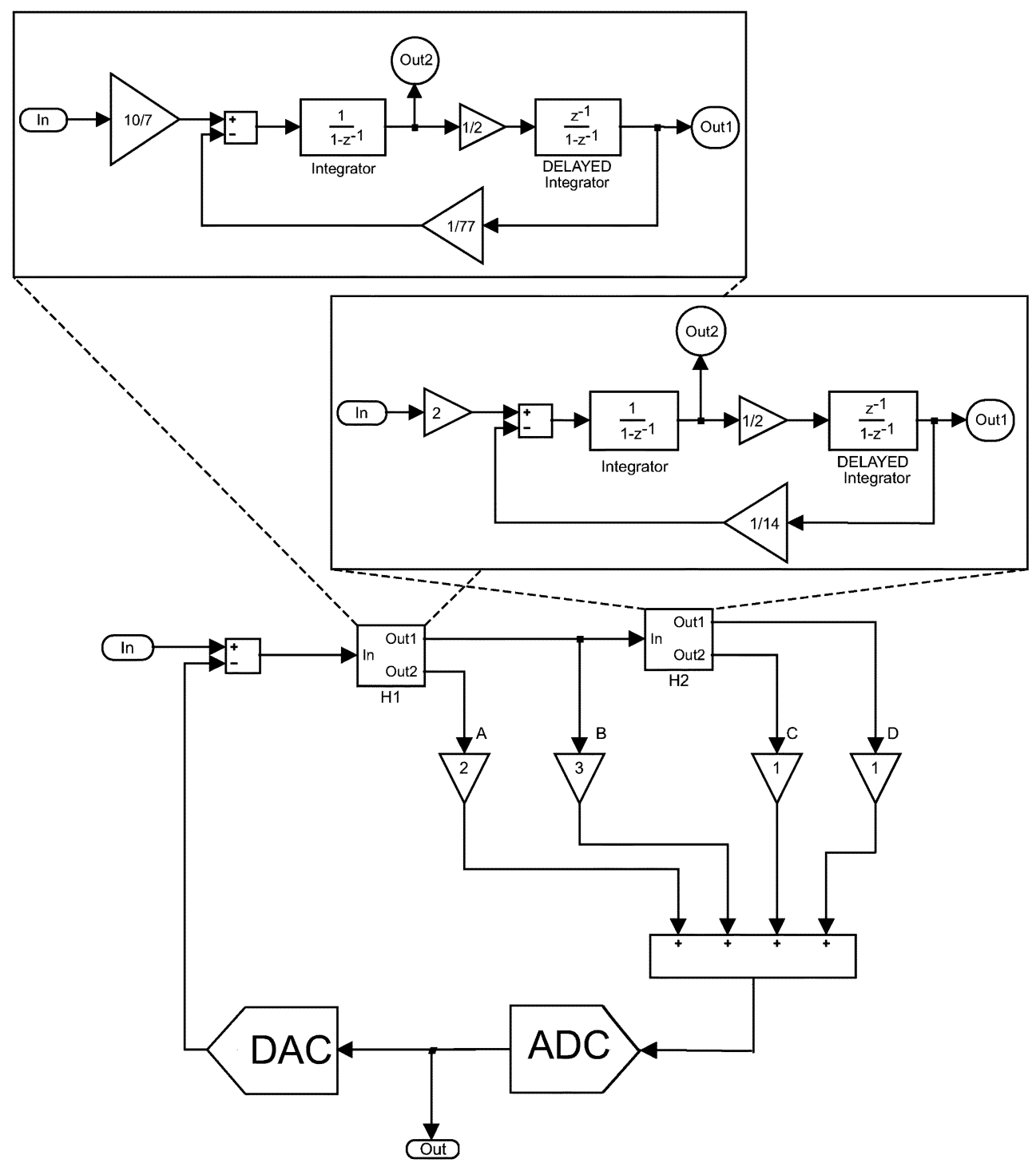

Fig. 4. Block diagram of one path of the proposed $\Sigma \Delta$ modulator.

outputs. The capacitors charged during phase $\Phi_{2}$ are connected in parallel and drive the comparator during phase $\Phi_{1}$ giving

$$
V_{x}=\frac{V_{A} 2 C+V_{B} 3 C+V_{C} C+V_{D} C-V_{\mathrm{ref}} C}{8 C+C_{p}} .
$$

The eight comparators of the quantizer use proper reference voltages $V_{\text {ref }} . C_{p}$ is the parasitic capacitance at the input of the comparator.

The passive solution adopted for implementing the adder causes an attenuation of the signal by at least a factor 8 (if $C_{p}$ is negligible). Moreover, the circuit is sensitive to the comparator offset. The offset mismatches cause nonlinearity and possibly nonmonotonicy. Thus, in order to avoid these problems, we use a simple gain stage with autozero in front of the comparators, as shown in Fig. 7.

\section{BUILDING BLOCKS}

The ADC has been fabricated using a $0.35-\mu \mathrm{m}$ double-poly triple-metal $3.3 \mathrm{~V}$ CMOS technology. The sampling input switch is an nMOS device with "bootstrapped" control. The bootstrap circuit maintains almost constant the $V_{\mathrm{gs}}$ in the on phase thus leading to a constant on-resistance [12].

The operational amplifier used is based on a fully differential two-stage topology (Fig. 8). The first stage is an $n$-channel telescopic cascode and the second stage is an $n$-channel differential pair with active load [13]. Both the first and second stages use a SC common-mode feedback. Miller capacitors with zero nulling resistor ensure stability. Behavioral simulations of the modulator have shown that at least a dc gain of $80 \mathrm{~dB}$, a gain-bandwidth product of $325 \mathrm{MHz}$ and a slew-rate of $400 \mathrm{~V} / \mu$ s are required to guarantee the $\Sigma \Delta$ modulator performance (considering also path mismatches). Table II summarizes the simulated performance of the amplifier. The achieved values are larger than the specifications, in order to ensure proper operation also in the process and temperature corners.

Fig. 9 shows the overall clock distribution scheme. A $280-\mathrm{MHz}$ sinusoidal clock is converted to a square waveform and its frequency divided by 4 , thus obtaining the $70-\mathrm{MHz}$ square wave main clock. The main clock is also shifted three 


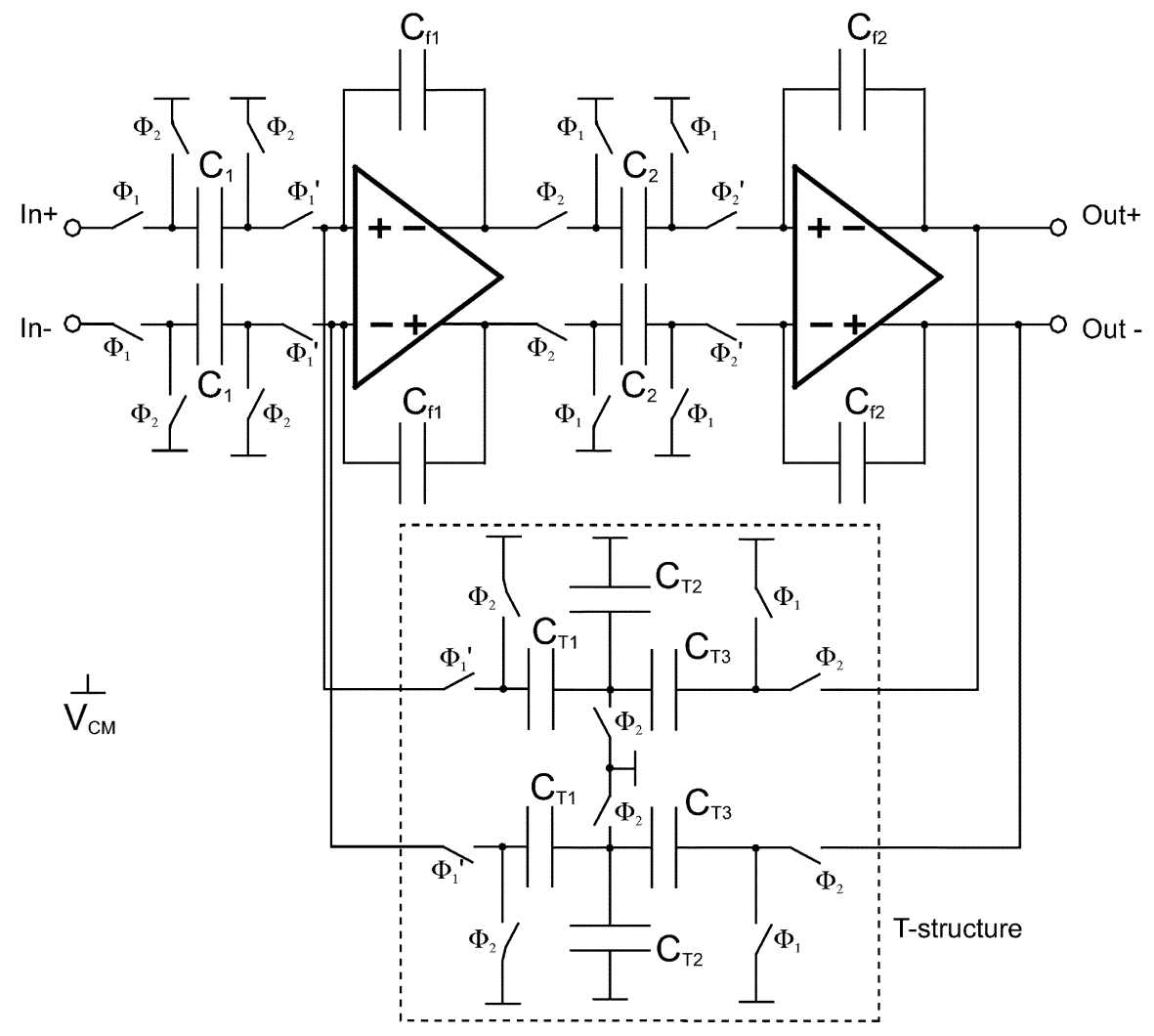

Fig. 5. SC implementation of the resonator.

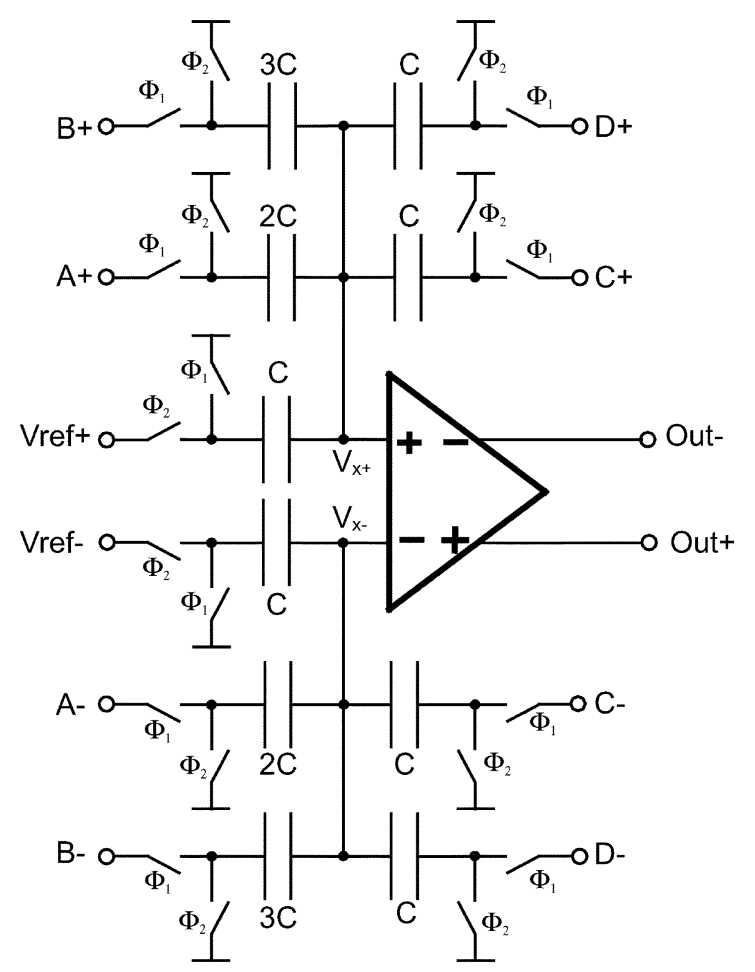

Fig. 6. SC implementation of the quantizer.

times by $90^{\circ}$, producing the clocks for the four modulator paths. They are the basis for the controls required by each modulator path. Simulations of the entire phase generator, including also the bonding wires and package models, gives a clock jitter that goes from 335 fs to $940 \mathrm{fs}$ depending on the considered

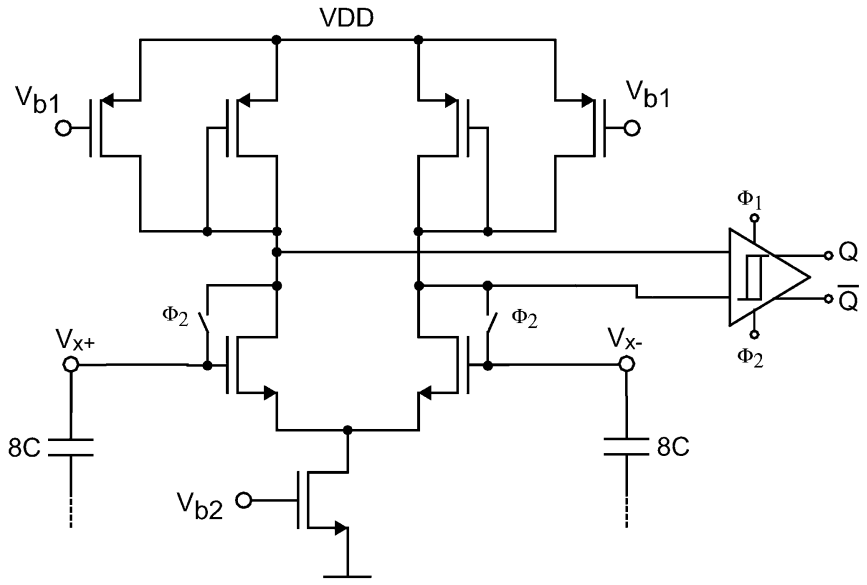

Fig. 7. Comparator structure: Gain stage followed by a latch.

phase. The lowest jitter phases are the ones used to drive the bootstrapped input switches.

The comparator (Figs. 6 and 7) consists of a gain stage followed by a latch [14]. During phase $\Phi_{2}$ the latch is reset while the gain stage is closed in unit gain configuration. The p-channel current source enhance the gain without requiring long p-channel diode-connected elements. Also during phase $\Phi_{2}$ the eight capacitors of the passive adder sample the offset of the gain stage. During $\Phi_{1}$ the capacitors sample the outputs $A, B, C, D$ of the resonators (Fig. 4) as well as the reference voltages with the proper weight (Fig. 6) and gain stage settles to the required value. Finally, on the falling edge of $\Phi_{1}$ the latch is released. This solution strongly reduces the offset as well as the kickback from the latch. 


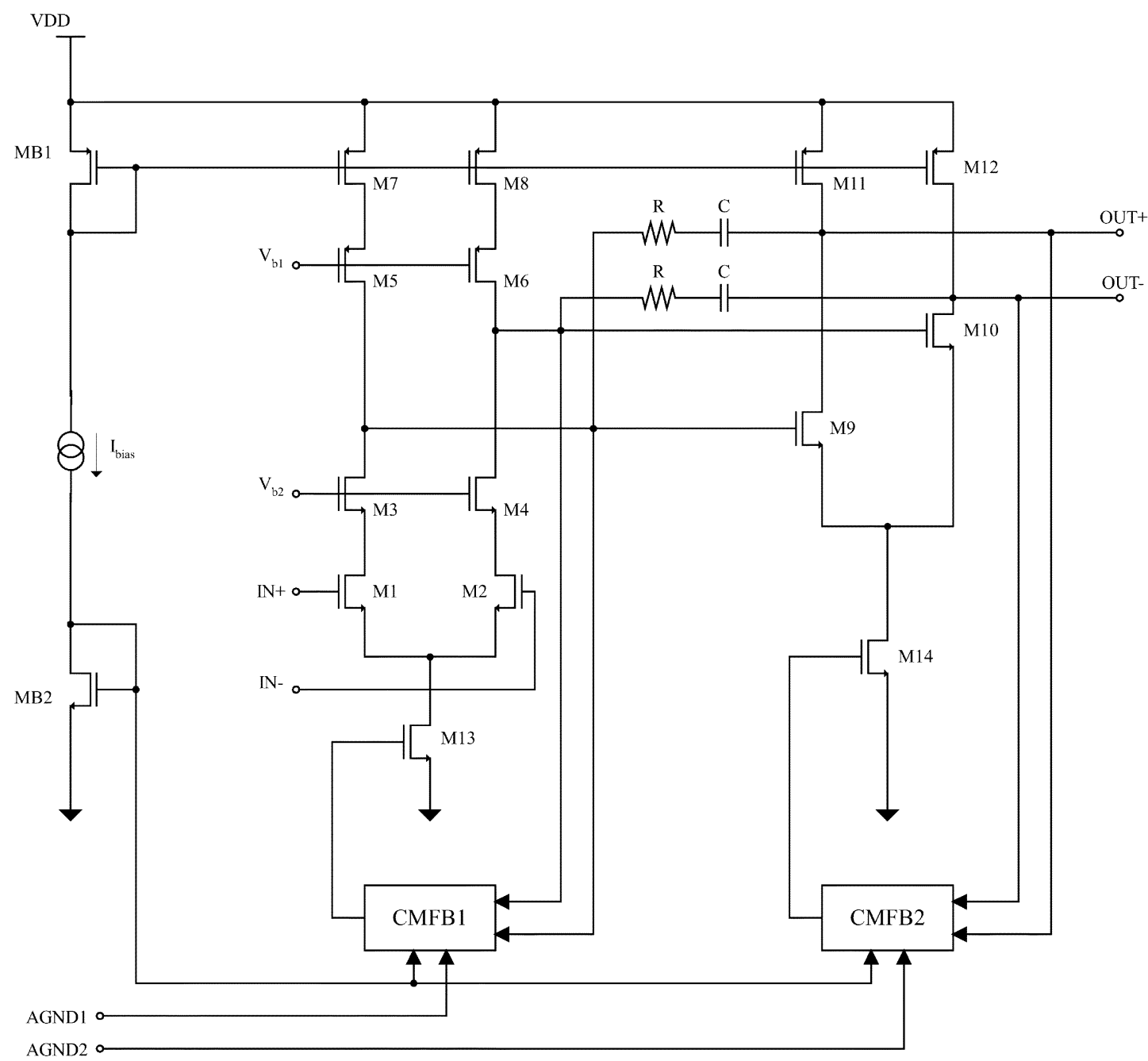

Fig. 8. Schematic diagram of the fully differential operational amplifier.

TABLE II

SiMULATEd PERFORMANCE OF OPERATIONAL AMPLIFIER

\begin{tabular}{|l|c|}
\hline \multicolumn{1}{|c|}{ Parameter } & Value \\
\hline \hline DC gain & $95 \mathrm{~dB}$ \\
\hline Gain-bandwidth product & $550 \mathrm{MHz}$ \\
\hline Phase margin & $53^{\circ}$ \\
\hline Slew-rate & $796 \mathrm{~V} / \mu \mathrm{s}$ \\
\hline Settling time (14 bit) & $4.1 \mathrm{~ns}$ \\
\hline Current consumption & $8.9 \mathrm{~mA}$ \\
\hline Input referred noise $(0-70 \mathrm{MHz})$ & $13 \mu \mathrm{V}$ \\
\hline
\end{tabular}

The DAC is an SC structure at the input of the integrators. It generates a midtread input-output characteristic with levels symmetrical with respect to the analog zero. Fig. 10 shows the structure of the DAC. During $\Phi_{2}$, all the capacitors are discharged, while during $\Phi_{1}$ depending on the modulator outputs some capacitors are connected to the positive or negative reference voltage. The capacitors which are not used are connected to ground. The mismatch among capacitors is corrected a using the noise-shaped dynamic element matching (DEM) technique [15]. By using this technique, it is possible to apply the noise-shaping principle to the errors introduced by

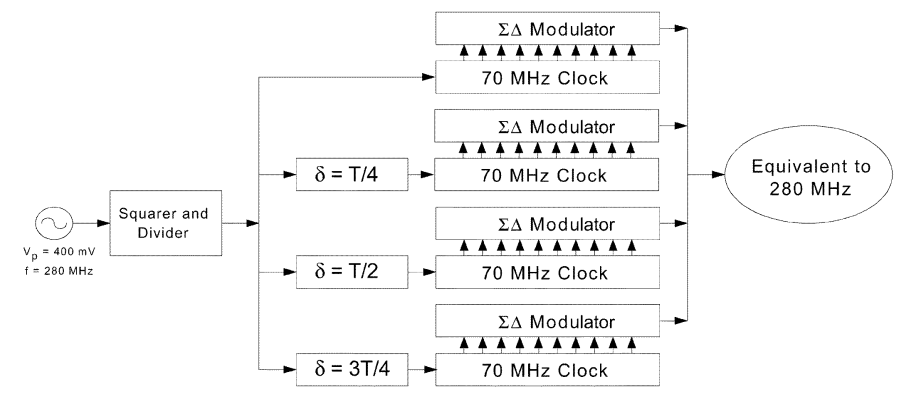

Fig. 9. General block diagram of the clock distribution scheme for the modulator.

capacitor mismatches. In our design, this known technique has been adapted to work with bipolar signals [16], [17].

Noise in the reference voltages, if larger than $5 \mathrm{mV}$, compromises the overall performance. The problem is mitigated by using low-noise reference buffers.

\section{EXPERIMENTAL RESULTS}

The micrograph of the fabricated chip is shown in Fig. 11 The area of the complete modulator and an extra single-path for testing purposes is $4 \times 6.8 \mathrm{~mm}^{2}$. The actual four-path $\Sigma \Delta$ modulator area is $20 \mathrm{~mm}^{2}$. The chip uses a CQFP package. 


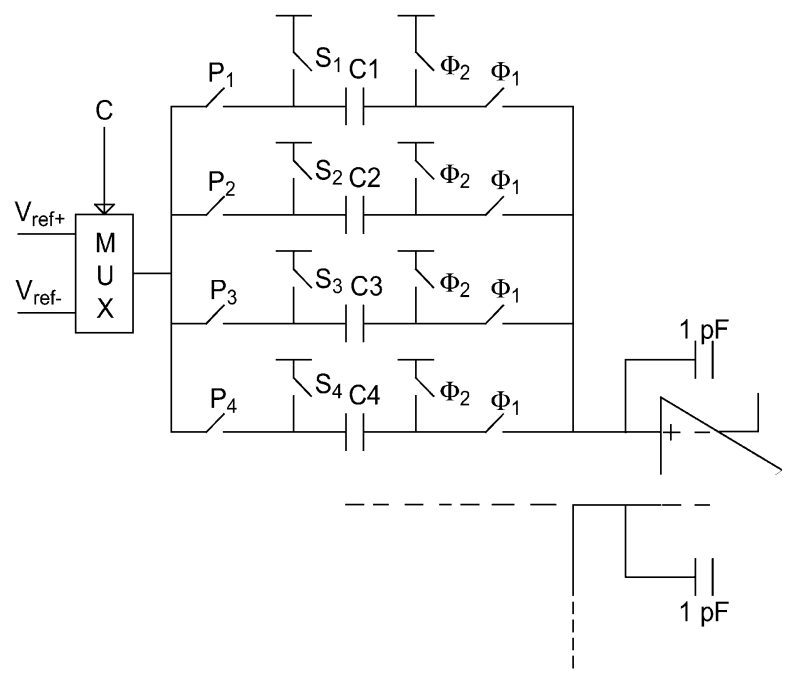

Fig. 10. SC implementation of the DAC.

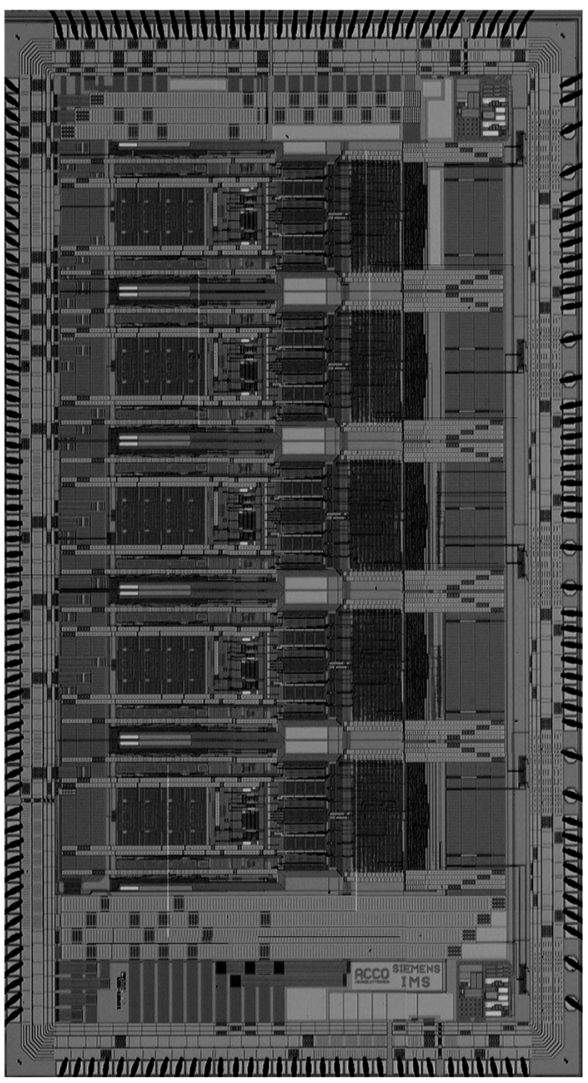

Fig. 11. Microphotograph of the four-path $\Sigma \Delta$ modulator chip prototype.

The chip has been extensively characterized. The PCB used for the testing employs very low-noise high-linearity input buffer to obtain with a feedback scheme the single-ended to double-ended conversion. The use of an RF transformer is also possible but the solution was not used because it causes higher noise and masks the circuit performance. Moreover, the board includes low-noise circuitry for driving the internal reference buffers.

The output spectrum of the four-paths path $\Sigma \Delta$ modulator is shown in Fig. 12. The clock frequency is $280 \mathrm{MHz}$ and the input signal amplitude is $56 \mathrm{mV}$ peak-to-peak differential at 69.95
TABLE III

PERFormanCE SUMMARY OF FOUR-PATH $\Sigma \Delta$ MODUlator

\begin{tabular}{|l|c|}
\hline \multicolumn{1}{|c|}{ Parameter } & Value \\
\hline \hline Maximum sampling frequency & $280 \mathrm{MHz}$ \\
\hline Maximum signal bandwidth & $4.375 \mathrm{MHz}$ \\
\hline DR @ $4.375 \mathrm{MHz}$ & $72 \mathrm{~dB}$ (corresponding to 12 bits) \\
\hline Maximum differential input signal & $1 \mathrm{~V}_{\mathrm{pp}}$ \\
\hline SFDR & $>69 \mathrm{~dB}$ \\
IMD3@ $650 \mathrm{mV}_{\mathrm{pp}}$ & $<-60 \mathrm{dBc}$ \\
\hline Power supply voltage & $3.3 \mathrm{~V}$ \\
\hline Power consumption & $480 \mathrm{~mW}$ \\
\hline
\end{tabular}

TABLE IV

COMPARISON OF SOME PREVIOUSLY PUBLISHED WIDE-BAND ADCs

\begin{tabular}{|c|c|c|c|c|c|c|c|}
\hline Paper & $\begin{array}{c}\text { Bandwidth } \\
\text { [MHz] }\end{array}$ & $\begin{array}{c}I F \\
{[M H z]}\end{array}$ & $\begin{array}{l}D R \\
{[d B]}\end{array}$ & $\begin{array}{c}S F D R \\
{[d B]}\end{array}$ & $\begin{array}{c}f_{c k} \\
{[M H z]}\end{array}$ & $\begin{array}{c}P \\
{[m W]}\end{array}$ & Technology \\
\hline This Work & 4.375 & 70 & 72 & 69 & 280 & 480 & $0.35 \mu \mathrm{m}$ \\
\hline ISSCC $2002|18|$ & 3.84 & 60 & 48 & 60 & 80 & 38 & $0.35 \mu \mathrm{m}$ \\
\hline CICC 2003 [19] & 2.5 & 0 & 82 & 100 & 40 & 495 & $0.35 \mu \mathrm{m}$ \\
\hline ESSCIRC 2003 [20| & 15 & 0 & 62 & - & 300 & 70 & $0.13 \mu \mathrm{m}$ \\
\hline ISSCC $2004|21|$ & 2.5 & 40 & 72 & 86 & 120 & 150 & $0.18 \mu \mathrm{m}$ \\
\hline VLSI $2004|5|$ & 1.25 & 60 & 52 & - & 240 & 37 & $0.35 \mu \mathrm{m}$ \\
\hline ISSCC 2005 [22] & 20 & 0 & 75 & 87 & 80 & 240 & $0.18 \mu \mathrm{m}$ \\
\hline
\end{tabular}

MHz. No spurious tones due to offset and gain mismatches among the different paths can be observed, thank to the careful design and to the relatively large noise floor allowed. The SNDR as a function of the input signal amplitude for the modulator is shown in Fig. 13. The DR achieves $72 \mathrm{~dB}$ peak (12 bits). The peak SNDR is achieved with an input signal of $-13 \mathrm{~dB}$. For larger amplitudes the performance is degraded by quantization noise tones due to the conditional stability of the modulator. The maximum signal amplitude was sacrificed in the design phase to guarantee fast overload recovery (fast resume of normal operation when the input signal becomes momentarily larger than $-13 \mathrm{~dB})$. This is an important feature in telecommunication systems and is worth a reduction of the maximum input signal.

Fig. 14 shows the output spectrum for an intermodulation test. The input signal consists of two tones at $69.9 / 70.1 \mathrm{MHz}$ and amplitude $650-\mathrm{mV}$ peak-to-peak differential each. The intermodulation products are $60 \mathrm{~dB}$ below the signal tones, corresponding to an $\mathrm{HD}_{3}$ of $-69 \mathrm{dBc}$ and hence leading to an equivalent SFDR of $69 \mathrm{~dB}$.

The total power consumption is $480 \mathrm{~mW}$ with $3.3-\mathrm{V}$ supply voltage, as expected. The features of the modulator are summarized in Table III.

Table IV compares the performance of this design with other published wide-band ADCs. Observe that the previous art either features limited bandwidth or smaller DR when compared to this design. Only power/band ineffective (for the considered application) low-pass solutions achieve performance comparable with the obtained results or better. For example, the solution reported in [20] requires a slew-rate in the operational amplifiers 


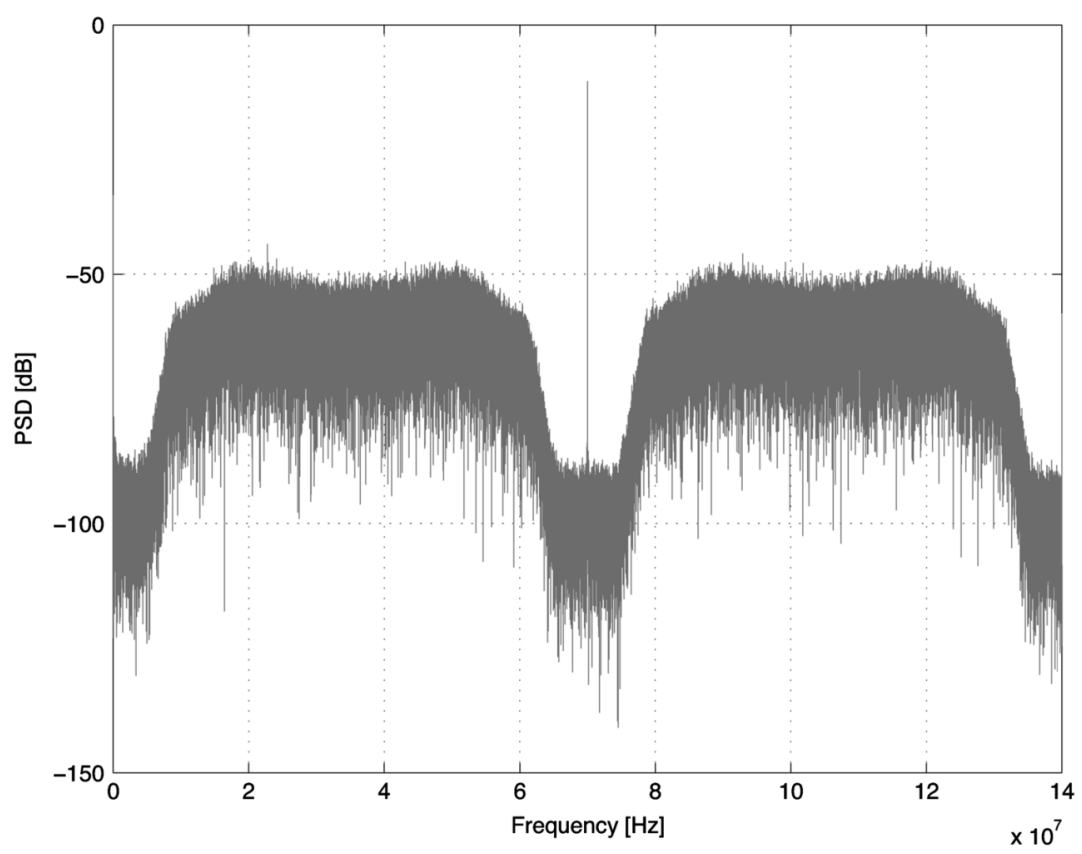

Fig. 12. Output spectrum of the four-path $\Sigma \Delta$ modulator obtained with clock frequency of $280 \mathrm{MHz}$, input signal amplitude of $56 \mathrm{mV}$ peak-to-peak differential, and input signal frequency of $69.95 \mathrm{MHz}$.

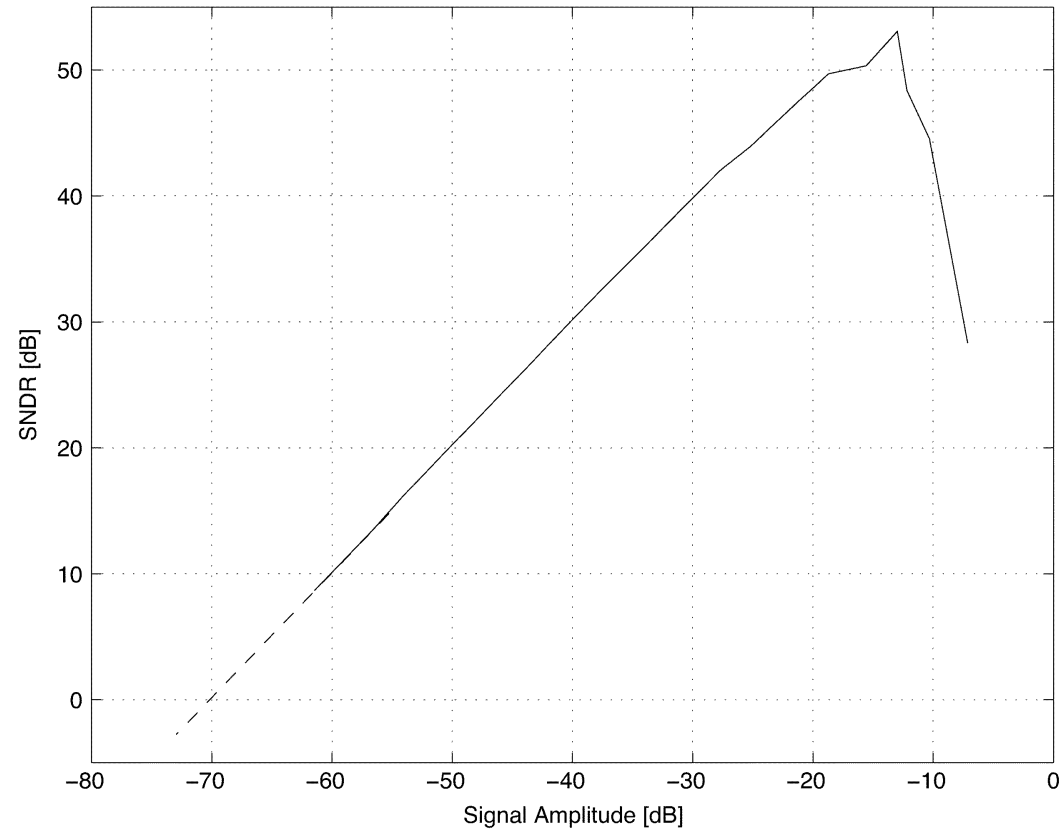

Fig. 13. SNDR as a function of the input signal amplitude of the four-paths $\Sigma \Delta$ modulator obtained with clock frequency of $280 \mathrm{MHz}$ and input signal frequency of $69.95 \mathrm{MHz}$.

$15 / 70$ lower than the proposed modulator and uses a technology scaled by about 0.4 . Therefore, a rough comparison with homogeneous features would require the multiplication of the power consumption of [20] by a factor of about 12 .

\section{CONCLUSION}

Bandpass modulators are a promising solution for the conversion of high-speed telecommunication signals. In this paper, we presented a four-path time-interleaved bandpass $\Sigma \Delta$ modulator architecture, which allows us to relax the speed require- ments of each path, thus making less critical the design of the building blocks, namely operational amplifiers, switches, ADC and DAC. The proposed architecture is demonstrated through the implementation of a four-path bandpass $\Sigma \Delta$ modulator operating with an overall clock frequency of $280 \mathrm{MHz}$ (each path works at $70 \mathrm{MHz}$ ). The achieved performance (DR of $72 \mathrm{~dB}, 12$ bits of resolution, with a signal bandwidth of $4.375 \mathrm{MHz}$ centered around an intermediate frequency of $70 \mathrm{MHz}$, SFDR of $69 \mathrm{~dB}$ ), satisfies the challenging requirements of $3 \mathrm{G}$ multicarrier wireless receivers. 


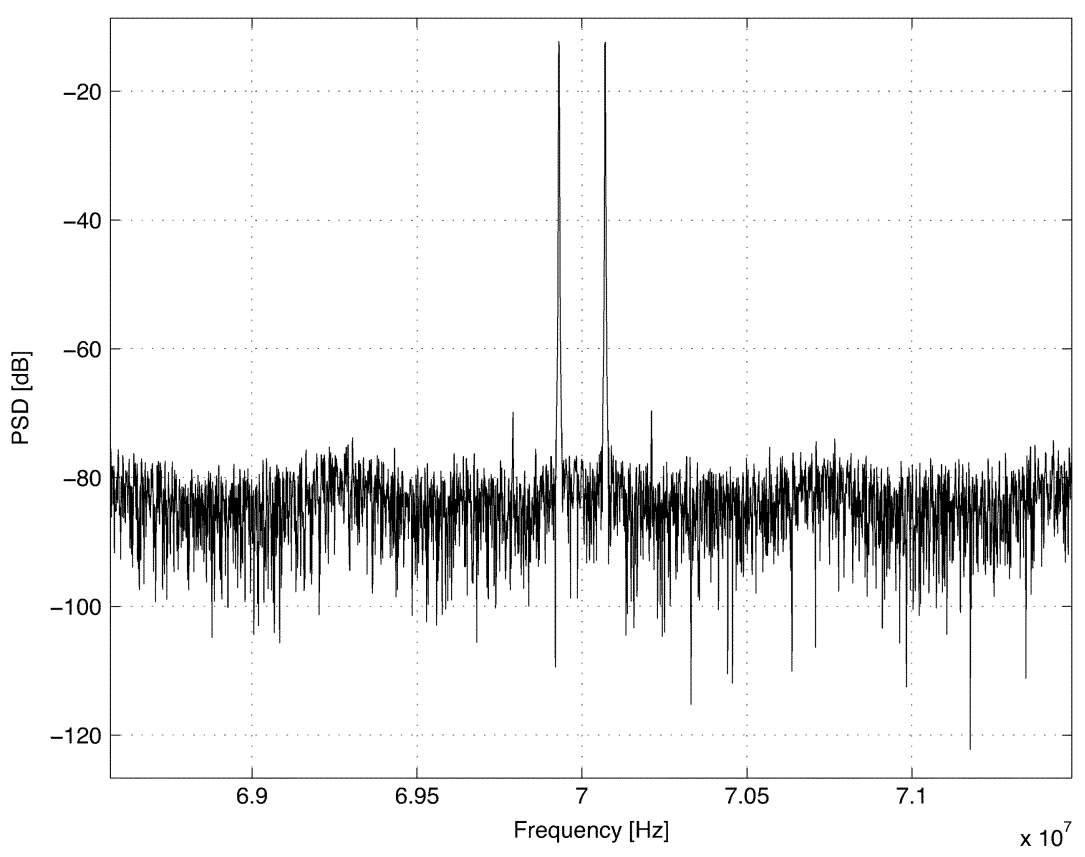

Fig. 14. Output spectrum of the four-paths $\Sigma \Delta$ modulator obtained in the intermodulation test with clock frequency of 280-MHz, input signal amplitude of 650-mV peak-to-peak differential and frequency the two tones of 69.9/70.1 $\mathrm{MHz}$.

\section{ACKNOWLEDGMENT}

The authors wish to thank R. Pantaleoni and M. Orlandi for the measurements and F. Francesconi, S. Brigati, and M. Poletti for the layout.

\section{REFERENCES}

[1] J. Khoury and T. Hai, "Data converters for communication systems," IEEE Commun. Mag., vol. 36, no. 10, pp. 113-117, Oct. 1998.

[2] A. M. Badá, G. Bucci, M. Donati, M. Maddiotto, M. Politi, and C. Savazzi, "Multicarrier software radio transceiver for mobile radio system," in Proc. ICT'99, Cheju Is, Korea, Jun. 1999.

[3] A. M. Badá and M. Donati, "The software radio technique applied to the RF front-end for cellular mobile systems," in Proc. Workshop on Software Radio: Technologies and Services, Isola d'OElba, Italy, Sep. 2000.

[4] S. Barzajani and W. M. Snelgrove, "A 160-MHz fourth-order doublesampled SC bandpass sigma-delta modulator," IEEE Trans. Circuits Syst. II, Analog Digit. Signal Process., vol. 45, no. 5, pp. 547-555, May 1998.

[5] V. S. L. Cheung and H. C. Luong, "A 3.3-V 240-MS/s CMOS bandpass $\Delta \Sigma$ modulator using a fast-settling double-sampling SC filter," in Dig. Tech. Papers IEEE Symp. VLSI Circuits (VLSI'04), Jun. 2004, pp. 84-87.

[6] W. Black and D. Hodges, "Time interleaved converter array," IEEE J. Solid-State Circuits, vol. SC-15, no. 6, pp. 1022-1029, Dec. 1980.

[7] S. Norsworthy, R. Schreier, and G. Temes, Delta-Sigma Data Converters-Theory, Design and Simulation. Piscataway, NJ: IEEE Press, 1997.

[8] V. Ferragina, A. Fornasari, U. Gatti, P. Malcovati, and F. Maloberti, "Gain and offset mismatch calibration in time-interleaved multipath A/D sigma-delta modulators," IEEE Trans. Circuits Syst. I, Reg. Papers, vol. 51, no. 12, pp. 2365-2373, Dec. 2004.

[9] CWTS, IMT2000-CWTS WG1Technical Specification TS C402 CWTS, ITU-R Rec.

[10] TD-SCDMA Radio Transmission Technology, CATT, Ver. 1.0.0, Dec. 1999.

[11] S. Brigati, F. Francesconi, P. Malcovati, D. Tonietto, A. Baschirotto, and F. Maloberti, "Behavioral modeling of switched-capacitor sigma-delta modulators," IEEE Trans. Circuits Syst. I, Fundam. Theory Appl., vol. 50, no. 3, pp. 352-364, Mar. 2003.
[12] J. Steensgaard, "Bootstrapped low-voltage analog switches," in Proc. IEEE Int. Symp. Circuits Syst. (ISCAS'99), May 1999, vol. 2, pp. 29-32.

[13] Y. Geerts, A. Marques, M. Steyaert, and W. Sansen, "A 3.3-V, 15-bit, delta-sigma ADC with a signal bandwidth of $1.1 \mathrm{MHz}$ for ADSL Application," IEEE J. Solid-State Circuits, vol. 34, no. 7, pp. 927-936, Jul. 1999.

[14] G. M. Yin, F. Op't Einde, and W. Sansen, "A high-speed comparator with 8-b resolution," IEEE J. Solid-State Circuits, vol. 27, no. 2, pp. 208-211, Feb. 1992.

[15] R. Schreier and B. Zhang, "Noise-shaped multibit D/A converter employing unit elements," Electron. Lett., vol. 31, no. 20, pp. 1712-1713, Sep. 1995.

[16] V. Ferragina, A. Fornasari, U. Gatti, P. Malcovati, F. Maloberti, and L. Monfasani, "Use of dynamic element matching in a multi-path sigma-delta modulator," in Proc. IEEE Int. Symp. Circuits Syst. (ISCAS'04), May 2004, vol. 1, pp. 649-652.

[17] P. Rombouts and L. Weyten, "A study of dynamic element-matching techniques for 3-level unit elements," IEEE Trans. Circuits Syst. II, Analog Digit. Signal Process., vol. 47, no. 11, pp. 1177-1187, Nov. 2000.

[18] T. Salo, T. Hollman, S. Lindfors, and K. Halonen, "A dual-mode 80-MHz bandpass $\Delta \Sigma$ modulator for a GSM/WCDMA IF-receiver," in Dig. Tech. Papers IEEE Int. Solid-State Circuits Conf. (ISSCC'02), Feb. 2002, pp. 218-219.

[19] Y.-I. Park, S. Karthikeyan, W. M. Koe, Z. Jiang, and T.-C. Tan, "A 16-bit, 5-MHz multi-bit sigma-delta ADC using adaptively randomized DWA," in Proc. IEEE Custom Integr. Circuits Conf. (CICC'03), Sep. 2003, pp. 115-118.

[20] A. D. Giandomenico, S. Paton, A. Wiesbauer, L. Hernandez, T. Potscher, and L. Dorrer, "A $15-\mathrm{MHz}$ bandwidth sigma-delta ADC with 11 bits of resolution in $0.13 \mu \mathrm{m}$ CMOS," in Proc. Eur. Solid-State Circuits Conf. (ESSCIRC'03), Sep. 2003, pp. 233-236.

[21] F. Ying and F. Maloberti, "A mirror image free two-path bandpass $\Sigma \Delta$ modulator with $72 \mathrm{~dB}$ SNR and $86 \mathrm{~dB}$ SFDR," in Dig. Tech. Papers IEEE Int. Solid-State Circuits Conf. (ISSCC'04), Feb. 2004, pp. 84-85.

[22] A. Bosi, A. Panigada, G. Cesura, and R. Castello, "An 80-MHz 4x oversampled cascaded $\Delta \Sigma$-pipelined ADC with $75 \mathrm{~dB}$ DR and 87 dB SFDR," in IDig. Tech. Papers IEEE Int. Solid-State Circuits Conf. (ISSCC'05), Feb. 2005, pp. 174-175. 


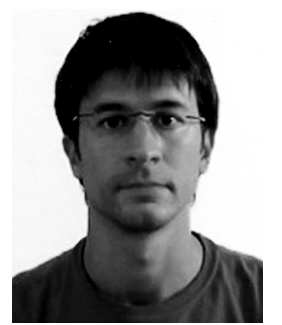

Gabriele Bernardinis was born in Udine, Italy, in 1976. He received the Laurea and Ph.D. degrees in electrical engineering and computer science from the University of Pavia, Pavia, Italy, in 2001 and 2004, respectively.

He was a Summer Intern with Texas Instruments, Dallas, TX, in 2000, working on a new approach for mismatch correction in time-interleaved pipeline analog-digital converters (ADCs), and a Summer Intern with National Semiconductor, Santa Clara, CA, in 2003, working on a high-speed high resolution ADCs. During his studies, he worked on CMOS oversampling and Nyquist-rate ADCs, focusing on time-interleaved pipeline and sigma-delta topologies. He joined International Rectifier's Rotation Program, El Segundo, CA, in 2004.

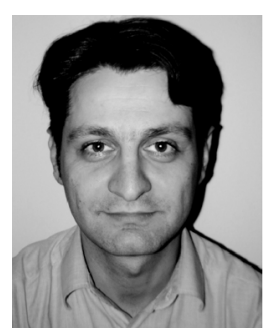

Fausto Borghetti was born in Verona, Italy, in 1974. $\mathrm{He}$ received the Laurea and Ph.D. degrees in electronics and computer science from the University of Pavia, Pavia, Italy, in 2000 and 2005, respectively.

In 2005, he joined the Integrated Optical Sensor Group, Istituto Trentino di Cultura / Istituto Ricerca Scientifica e Tecnologica (ITC-IRST), Povo, Italy. His research interests are in high-performance analog-digital converters, DC/DC converters and CMOS image sensors.

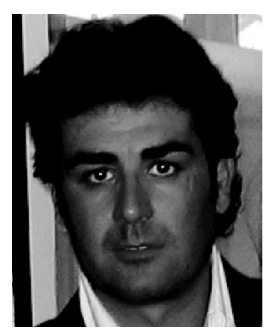

Vincenzo Ferragina was born in Catanzaro, Italy, in 1973. He received the Laurea degree in electronic engineering from the University of Pavia, Pavia, Italy, in 2002 , where he is currently working toward the Ph.D. degree.

His research interests include digital design, mixed analog-digital design, and analysis of substrate noise in mixed analog-digital integrated circuits. Presently, he is involved in the design of a 8 -bit $250-\mathrm{MHz}$ pipeline analog-digital converter (ADC). Besides, he is working on the digital back-end of an application-specific integrated circuit for reading-out GaAs pixel arrays devoted to $\mathrm{X}$-ray spectroscopy and imaging in the $0.5-\mathrm{keV}$ energy range.

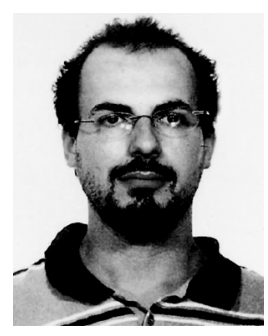

Andrea Fornasari was born in Piacenza, Italy, on November 19, 1976. He received the Laurea and Ph.D. degrees from the University of Pavia, Pavia, Italy in 2000 and 2004, respectively.

In this period, his main field of interest was the design of analog-digital converters (ADCs) and the study of digital calibration of circuital non idealities, receiving a patent in this area. In 2004, he joined the Standard Linear Group of Austriamicrosystems, Pavia, Italy, where he is involved in the design of very low-power analog blocks.

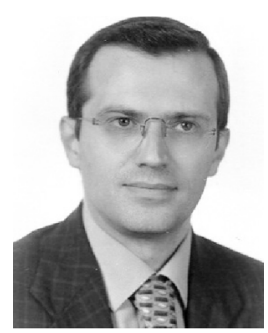

Umberto Gatti (M'91) was born in Pavia, Italy, in 1962. He received the Laurea degree in electronic engineering (summa cum laude) and the Ph.D. degree in electronics and information engineering from the University of Pavia, Pavia, Italy, in 1987, and 1992, respectively.

From 1993 to 1999, he worked in the Central R\&D Laboratory, Italtel S.p.A., Milan, Italy, as an ASIC Designer. In 1999, he joined the Development Technologies Laboratory, Siemens ICN S.p.A. (now, Siemens S.p.A.), Milan, Italy, where he is currently a Senior Design Engineer. His research interests are in the area of CMOS and BiCMOS integrated circuits for analog and mixed-signal processing and of signal integrity issues in printed circuit boards (PCBs). He was involved in the design of high-speed data converters, while presently he is involved in the study of broadband wireless transceivers and in the signal integrity analysis of complex PCB for high-performance telecom applications.

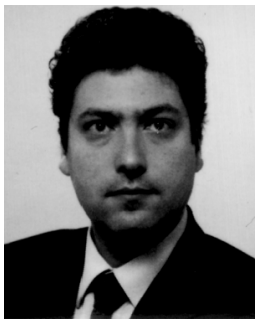

Piero Malcovati (S'94-M'95-SM'02) was born in Milano, Italy, in 1968. He received the Laurea degree (summa cum laude) in electronic engineering and the $\mathrm{Ph} . \mathrm{D}$. degree in electrical engineering from University of Pavia, Pavia, Italy, and the Physical Electronics Laboratory (PEL), Federal Institute of Technology, Zurich (ETH Zurich), Switzerland, in 1991, and 1996, respectively.

From 1996 to 2001, he was an Assistant Professor in the Department of Electrical Engineering, University of Pavia. From 2002, he is an Associate Professor of Electrical Measurements in the same institution. His research activities are focused on microsensor interface circuits and high performance data converters. He has authored and co-authored more than 20 papers in International Journals, more than 50 presentations at International Conferences (with published proceedings), four book chapters, and three industrial patents.

Dr. Malcovati was Guest Editor for the Journal of Analog Integrated Circuits and Signal Processing for the special issue on IEEE ICECS 1999. He served as Special Session Chairman for the IEEE ICECS 2001 Conference, as Secretary of the Technical Program Committee for the ESSCIRC 2002 Conference, and as Technical Program Chairman of the IEEE PRIME 2006 Conference. He was and still is member of the Scientific Committees for several International Conferences, including ESSCIRC, DATE, and PRIME. He is associate editor of the Journal of Circuits, Systems, and Computers.

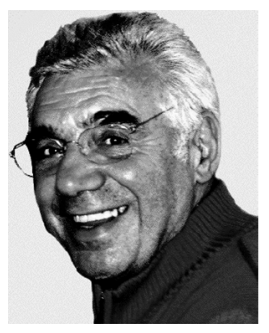

Franco Maloberti (SM'87-F'97) received the Laurea degree in physics (summa cum laude) from the University of Parma, Parma, Italy, in 1968 and the Dr. Honoris Causa degree in electronics from the Instituto Nacional de Astrofisica, Optica y Electronica (Inaoe), Puebla, Mexico in 1996.

He was a Visiting Professor at the Physical Electronics Laboratory (PEL), Federal Institute of Technology, Zurich (ETH Zurich), Switzerland, in 1993 and at the The Electronics Laboratory, École Polytechnique Fédérale de Lausanne (EPFL-LEG), Lausanne, Switzerland, in 2004. He was Professor of Microelectronics and Head of the Micro Integrated Systems Group University of Pavia, Pavia, Italy, and the TI/J.Kilby Analog Engineering Chair Professor at the Texas A\&M University, Houston. He was also the Distinguished Microelectronic Chair Professor at University of Texas at Dallas. Currently he is Professor at the University of Pavia. His professional expertise is in the design, analysis, and characterization of integrated circuits and analog digital applications, mainly in the areas of switched-capacitor circuits, data converters, interfaces for telecommunication and sensor systems, and computer-aided design for analog and mixed analog-digital (A/D) design. He has been responsible at both technical and management levels for many research programs including ten ESPRIT projects and has served the European Commission as ESPRIT Projects' Evaluator, Reviewer and as European Union expert in many European Initiatives. He served the Academy of Finland on the assessment of electronic research in Academic institutions and on the research programs' evaluations. He served the National Research Council of Portugal on a Board for the research activity assessment of Portuguese Universities. He has written more than 300 published papers, three books and holds 17 patents.

Dr. Maloberti was the recipient of the XII Pedriali Prize in 1992 for his technical and scientific contributions to national industrial production, co-recipient of the 1996 Institute of Electrical Engineers (U.K.) Fleming Premium for the paper "CMOS Triode Transistor Transconductor for high-frequency continuous time filters." He was Vice-President, Region 8, of the IEEE Circuit and Systems Society (CAS-S) from 1995 to 1997 and an Associate Editor of IEEE TRANSACTIONS ON CiRCUITS AND SYSTEM-II: ANALOG Digital Signal PROCESSING, in 1998. He received the 1999 IEEE CAS-S Meritorious Service Award, the 2000 CAS-S Golden Jubilee Medal, and the IEEE Millenium Medal. He was the President of the IEEE Sensor Council (2002-2003) and a member of the BoG of the IEEE CAS-S (2003-2005). He is currently an Associate Editor of the IEEE TRANSACTIONS ON CIRCUITS AND SYSTEM-II: EXPRESS BRIEFS, and member of the Editorial Board of Analog Integrated Circuits and Signal Processing. 